Revue d'histoire de l'Amérique française

REVUE D.HISTOIRE DE L'AMÉRIQUE FRANÇAISE

DELANGLEZ, JEAN, s.j., Louis Jolliet, Vie et Voyages

(1645-1700) Les Études de l'Institut d'Histoire de l'Amérique française, 1950.

\title{
Lionel Groulx
}

Volume 4, numéro 1, juin 1950

URI : https://id.erudit.org/iderudit/801620ar

DOI : https://doi.org/10.7202/801620ar

Aller au sommaire du numéro

Éditeur(s)

Institut d'histoire de l'Amérique française

ISSN

0035-2357 (imprimé)

1492-1383 (numérique)

Découvrir la revue

Citer ce compte rendu

Groulx, L. (1950). Compte rendu de [DELANGLEZ, JEAN, s.j., Louis Jolliet, Vie et Voyages (1645-1700) Les Études de l'Institut d'Histoire de l'Amérique française, 1950.] Revue d'histoire de l'Amérique française, 4(1), 117-118. https://doi.org/10.7202/801620ar d'utilisation que vous pouvez consulter en ligne.

https://apropos.erudit.org/fr/usagers/politique-dutilisation/ 


\section{LIVRES ET REVUES}

Delanglez, Jean, s.j., Louis Jolliet, Vie et Voyages (1645-1700) Los Études de l'Institut d'Histoire de l'Amérique française, 1950.

Nous annonçons plus loin la mise an librairie très prochaine du Louis Jolliet du Père Delanglez. L'un de nos directeurs, M. Guy Frégault, publiera ici-même, dans notre livraison de septembre, un article sur cet ouvrage de l'historien. En attendant ces pages, on pourra lire cette courte préface qui figure au début de ce nouveau Jolliet et qui en expose sommairement le caractère.

J'écris ce bout de préface parce que l'auteur l'a voulu. Le 12 mars 1949, je lui avais mandé: "Vous me parlez d'une préface. Croyez-vous que, pour un homme de votre réputation, une préface soit nécessaire?" Il me répondit quelques jours plus tard: "Je crois vraiment qu'il faut que vous écriviez un ou deux mots d'introduction. Cuique suum. Et il y a beaucoup du vôtre dans ces pages."

Le Père Delanglez se montrait ici trop généreux. M. Guy Frégault et moi-même avions tout simplement revu la traduction française de son Life and Voyages of Louis Jolliet. J'ai déjà expliqué, dans la Revue d'Histoire de l'A mérique fransaise (septembre 1949, p. 313316), comment le texte du présent ouvrage fut établi. Belge d'origine, mais de formation et de culture américaines, le Père Delanglez lisait facilement le français; il sav it en saisir les moindres nuances ou subtilités. Il avait perdu l'habitude de l'écrire. Il fut donc convenu qu'il ferait lui-même la traduction de son ouvrage et qu'il pourrait compter sur nos services pour une revision de son texte. Ce texte, nous l'avons simplement revu; nous n'y avons rien changé. Du reste, l'auteur eut le temps de revoir lui-même son manuscrit avant de nous le confier pour l'impression.

Faut-il voir en Louis Jolliet, Vie et Voyages, une adaptation ou une traduction de Life and Voyages of Louis Jolliet? Il convient d'opter pour une adaptation. Les positions ou conclusions de l'auteur restent 
les mêmes; la démonstration se déroule parfois de façon différente, s'enrichit souvent d'autres preuves ou docu'ments. L'édition française de son ouvrage, le Père Delanglez l'avait voulue, sur plusieurs points, plus travaillée, plus complète que l'anglaise. Ses préférences allaient à celle-là. Et c'est parce qu'il y tenait si fortement qu'il y a travaillé, dans les derniers jours de sa vie, avec une sorte de fièvre.

Est-il besoin d'insister sur la valeur de ce Louis Jolliet? Dans un éloge de l'historien qui fut son maître, M. Guy Frégault écrivait: "L'érudit renouvelle les questions qu'il aborde. Et les solutions qu'il trouve renouvellent elles-mêmes les perspectives d'une époque. Frontenac, La Salle, Jolliet, Cadillac: autour de ces personnages de premier plan, gravite toute une période de l'histoire de l'Amérique française. Il est désormais impossible de l'étudier sans dépouiller les livres et les articles de l'historien". On ne saurait mieux dire. L'Institut d'Histoire de l'Amérique française est heureux et fier d'ajouter à ses "Etudes" une grande œuvre d'histoire scientifique. Ceux qui la liront regretteront avec nous la disparition de ce magnifique travailleur parti en pleine force, qui aurait pu nous laisser, sur le passé de notre Amérique, de si fortes œuvres.

Lionel Groulx, ptre. 